\title{
Platelet count predicts prognosis in operable non-small cell lung cancer
}

\author{
DANGFAN YU ${ }^{1}$, BINGJIANG LIU ${ }^{1}$, LIZHEN ZHANG ${ }^{1}$ and KAIQI DU ${ }^{2}$ \\ Departments of ${ }^{1}$ Radiology and ${ }^{2}$ Thoracic Surgery, Zhejiang Provincial Corps Hospital, \\ Chinese People's Armed Police Force, Jiaxing, Zhejiang 314000, P.R. China
}

Received November 25, 2012; Accepted February 8, 2013

DOI: $10.3892 /$ etm.2013.1003

\begin{abstract}
Platelets play a significant role in cancer cell growth, progression and metastasis. However, in non-small cell lung cancer (NSCLC), the association between a patient's platelet count and prognosis has not previously been fully elucidated. The aim of the present study was to investigate the correlation between platelet count, patients' characteristics and prognosis in patients with NSCLC. A total of 510 NSCLC patients were enrolled in the present study. The median platelet count in the NSCLC patients was $203 \times 10^{9} / 1$ (95\% CI, 115-358x10 $\left./ 1\right)$. The median platelet count in T3 and T4 patients was significantly higher than that of T1 and T2 patients (median, 263x10 $/ 1$ and $253.5 \times 10^{9} / 1$ vs. $199.5 \times 10^{9} / 1$ and $196.5 \times 10^{9} / 1$, respectively; $\mathrm{P}<0.001$ ). The 3 -year cumulative overall survival (OS) probability was $75.3 \%$ for patients with normal platelet counts and $59.2 \%$ for patients with elevated platelet counts. When compared with the patients with normal platelet counts, the patients with elevated platelet counts had an increased risk of disease progression (HR, 1.568; 95\% CI, 1.015-2.453). Pre-operative platelet counts are a novel independent prognostic biomarker in operable NSCLC.
\end{abstract}

\section{Introduction}

Non-small cell lung cancer (NSCLC) is the most common malignant neoplasm worldwide (1), with metastasis and recurrence primarily responsible for mortality. The early identification of metastasis and recurrence provides patients with time to undergo salvage therapy in order to prolong their survival. Radical surgery has been the standard treatment for a number of decades. However, a large number of patients experience disease progression over a short time. Over the past decades, various studies have attempted to identify molecular biomarkers to predict the metastasis or recurrence of NSCLC $(2,3)$. Numerous promising biomarkers have been

Correspondence to: Dr Yu Dangfan, Zhejiang Provincial Corps Hospital, 16 Nanhu Road, Jiaxing, Zhejiang 314000, P.R. China E-mail: diadem1982@126.com

Key words: non-small cell lung cancer, platelet counts, prognosis evaluated as potential prognosis predictors, however, none of these have been demonstrated to be sufficiently effective for clinical use. The majority of these markers are somewhat controversial and inconclusive.

More recently, significant attention has been given to the association between malignancies and coagulation $(4,5)$. A hypercoagulability state is one of the signs of a more aggressive disease, while a thromboembolism is one of the major causes of mortality in cancer patients (6). Several studies have demonstrated that an elevated platelet count correlates with a poor prognosis in numerous types of solid cancer, including colorectal cancer $(7,8)$, esophageal carcinoma (9) and gastric cancer $(10,11)$. A prognostic significance between the platelet count and lung cancer has also been identified (12-17). However, the majority of these studies included small cell lung cancer and the samples were relatively small.

In the present study, a retrospective clinical analysis was designed for a total of 510 operable NSCLC patients to investigate the correlation between platelet count, patients' characteristics and prognosis.

\section{Patients and methods}

Patients and treatment. The present study enrolled 510 patients who had been diagnosed with NSCLC between 2006 and 2009 at Zhejiang Provincial Corps Hospital, China. All patients had their diagnoses freshly confirmed and had not had any previous treatment. The patients with the following characteristics were excluded from the present study: patients who had any coexisting or previous cancers other than NSCLC; patients with concomitant diseases suspected of increasing the serum platelet concentration, including severe hypertension, splenic disease and blood coagulation disorders; and patients who had taken aspirin or other acetylsalicylic acid drugs one month prior to the treatment. The study population had a median age of 60 years (range, 37-82 years). The patients comprised of $388(76.1 \%)$ males and $122(23.9 \%)$ females. Approval for the present study was obtained from the institutional review board of the Zhejiang Provincial Corps Hospital. All patients provided informed consent prior to undergoing surgery.

A lobectomy, bilobectomy or pneumonectomy was performed according to the location or size of the lung neoplasm in each patient. Systematic mediastinum lymph node dissection was also performed in each patient. In total, 
Table I. Association of platelet count with the parameters of patients with NSCLC.

\begin{tabular}{|c|c|c|c|c|c|c|}
\hline \multirow[b]{2}{*}{ Parameters } & \multirow[b]{2}{*}{ Patients, n (\%) } & \multicolumn{5}{|c|}{ Platelet count } \\
\hline & & Median (5th-95th percentile) & P-value & $\leq 300, \mathrm{n}^{\mathrm{b}}(\%)$ & $>300, \mathrm{n}^{\mathrm{b}}(\%)$ & P-value \\
\hline Gender & & & 0.312 & & & 0.074 \\
\hline Male & $388(76.1)$ & $204.5(112.8-373.5)$ & & $336(86.6)$ & $52(13.4)$ & \\
\hline Female & $122(23.9)$ & $198.5(117.6-352.5)$ & & $113(92.6)$ & $9(7.4)$ & \\
\hline Age (years) & & & 0.263 & & & 0.158 \\
\hline$<65$ & $353(69.2)$ & $206.0(117.0-373.6)$ & & $306(86.7)$ & $47(13.3)$ & \\
\hline$\geq 65$ & $157(30.8)$ & $193.0(103.6-357.1)$ & & $143(91.1)$ & $14(8.9)$ & \\
\hline Smoking & & & 0.338 & & & 0.094 \\
\hline Never & $156(30.6)$ & $199.0(115.6-345.5)$ & & $143(91.7)$ & $13(8.3)$ & \\
\hline Smoker & $354(69.4)$ & $205.5(114.0-378.3)$ & & $306(86.4)$ & 48 (13.6) & \\
\hline Histological type & & & 0.058 & & & 0.127 \\
\hline Squamous cell carcinoma & $253(49.6)$ & $215.0(117.7-361.0)$ & & $216(85.4)$ & 37 (14.6) & \\
\hline Adenocarcinoma & $232(45.5)$ & $196.0(107.0-376.1)$ & & $209(90.1)$ & $23(9.9)$ & \\
\hline Other & $25(4.9)$ & $192.0(121.0-299.5)$ & & $24(96.0)$ & $1(4.0)$ & \\
\hline Differentiation & & & 0.122 & & & 0.959 \\
\hline Well & $29(5.7)$ & $187.0(78.0-314.5)$ & & $26(89.7)$ & $3(10.3)$ & \\
\hline Moderately & $263(51.6)$ & $201.0(118.4-333.6)$ & & $231(87.8)$ & $32(12.2)$ & \\
\hline Poorly & $218(42.7)$ & $210.5(114.8-380.1)$ & & $192(88.1)$ & $26(11.9)$ & \\
\hline T stage & & & $<0.001^{\mathrm{a}}$ & & & $<0.001^{\mathrm{a}}$ \\
\hline $\mathrm{T} 1$ & $52(10.2)$ & $196.5(96.3-325.8)$ & & $47(90.4)$ & $5(9.6)$ & \\
\hline $\mathrm{T} 2$ & $362(71.0)$ & $199.5(117.0-331.4)$ & & $329(90.9)$ & $33(9.1)$ & \\
\hline $\mathrm{T} 3$ & $60(11.8)$ & $253.5(127.4-423.5)$ & & $43(71.7)$ & $17(28.3)$ & \\
\hline $\mathrm{T} 4$ & $36(7.1)$ & $263.0(148.1-406.1)$ & & $30(83.3)$ & $6(16.7)$ & \\
\hline $\mathrm{N}$ stage & & & $0.001^{\mathrm{a}}$ & & & $0.001^{\mathrm{a}}$ \\
\hline No & $270(52.9)$ & $192.0(107.1-312.5)$ & & $251(93.0)$ & $19(7.0)$ & \\
\hline N1 & $135(26.5)$ & $217.0(110.6-379.2)$ & & $114(84.4)$ & $21(15.6)$ & \\
\hline $\mathrm{N} 2$ & $105(20.6)$ & $221.0(117.3-395.3)$ & & $84(80.0)$ & $21(20.0)$ & \\
\hline Clinical stage & & & $<0.001^{\mathrm{a}}$ & & & $<0.001^{\mathrm{a}}$ \\
\hline I & $234(45.9)$ & $190.5(107.5-305.3)$ & & $221(94.4)$ & $13(5.6)$ & \\
\hline II & $128(25.1)$ & 220.5 (113.7-383.8) & & $107(83.6)$ & $21(16.4)$ & \\
\hline III & $148(29.0)$ & $225.0(115.9-387.2)$ & & $121(81.8)$ & 27 (18.2) & \\
\hline
\end{tabular}

${ }^{\mathrm{a}} \mathrm{P}<0.05$. NSCLC, non-small cell lung cancer; ${ }^{\mathrm{b}} \mathrm{x} 10^{9}$ cells $/ 1$.

203 patients were treated with adjuvant platinum-based chemotherapy, adjuvant radiotherapy or a combination of the two. Detailed information about the patient's characteristics and tumor histopathology were collected retrospectively from the medical records.

Platelet measurement. A blood sample was obtained by peripheral venous puncture, before breakfast 3 days prior to the surgery. A complete blood count was regularly taken and elevated platelet counts were defined as $>300 \times 10^{9} / 1$.

Follow-up. All patients received a standardized follow-up, occurring at 3-month intervals for two years, at 6-month intervals in the third year and yearly thereafter. An evaluation comprised a physical examination, complete blood count, chest computed tomography $(\mathrm{CT})$, brain magnetic resonance imaging
(MRI) and abdominal ultrasound. Local recurrence and distance metastasis were histologically confirmed whenever possible.

Statistical analysis. The Chi-square test was performed to evaluate the association between the clinicopathological variables and the platelet count. Disease-free survival (DFS) was defined from the date of the definitive surgery to the date of local or distant progression, mortality by any cause or the date of the last follow-up. Overall survival (OS) was calculated as the time from the pulmonary surgery to the time of mortality or censoring. Kaplan-Meier curves were used to estimate the distribution of DFS and OS and a two-sided log-rank test was performed to compare the difference between the survival curves. The Cox proportional hazards model was used with a backward selection method for the univariate and multivariate analyses. All factors with an effect on DFS and OS in the 
Table II. Multivariate analysis of DFS and OS rate.

\begin{tabular}{|c|c|c|c|}
\hline Parameter & HR & $95 \% \mathrm{CI}$ & P-value \\
\hline \multicolumn{4}{|l|}{ DFS } \\
\hline Age: $<65$ vs. $\geq 65$ years & 1.416 & $1.009-1.987$ & $0.044^{\mathrm{a}}$ \\
\hline Smoking: ever vs. never & 1.029 & $0.596-1.777$ & 0.919 \\
\hline T stage: $\mathrm{T} 1$ and 2 vs. T3 and 4 & 1.395 & $0.892-2.183$ & 0.145 \\
\hline N stage: N0 vs. N1-2 & 1.113 & $0.733-1.692$ & 0.615 \\
\hline Clinical stage: I, II vs. III & 1.325 & $0.817-2.147$ & 0.254 \\
\hline Platelet count: $\leq 300 \times 10^{9}$ vs. $>300 \times 10^{9}$ cells $/ 1$ & 1.568 & $1.015-2.453$ & $0.030^{\mathrm{a}}$ \\
\hline \multicolumn{4}{|l|}{ OS } \\
\hline Age: $<65$ vs. $\geq 65$ years & 1.795 & $1.170-2.755$ & $0.007^{\mathrm{a}}$ \\
\hline Smoking: ever vs. never & 1.367 & $0.868-2.154$ & 0.178 \\
\hline T stage: T1-2 vs. T3-4 & 1.157 & $0.668-2.004$ & 0.602 \\
\hline N stage: N0 vs. N1-2 & 1.436 & $0.843-2.444$ & 0.183 \\
\hline Clinical stage: I, II vs. III & 1.496 & $0.834-2.683$ & 0.176 \\
\hline Platelet count: $\leq 300 \times 10^{9}$ vs. $>300 \times 10^{9}$ cells $/ 1$ & 1.689 & $1.005-2.380$ & $0.017^{\mathrm{a}}$ \\
\hline
\end{tabular}

${ }^{a} \mathrm{P}<0.05$. DFS, disease-free survival; OS, overall survival; HR, hazard ratio; CI, confidence interval.
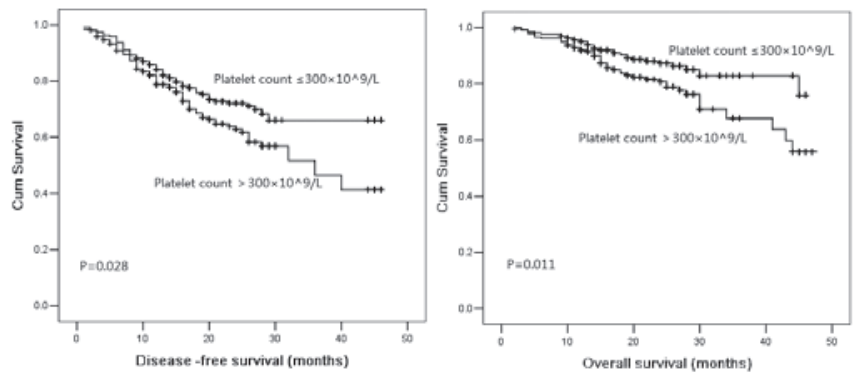

Figure 1. (A) Kaplan-Meier disease-free survival (DFS) curves and (B) overall survival (OS) curves according to platelet count; patients with normal platelet counts showed longer DFS and OS times.

univariate analysis $(\mathrm{P} \leq 0.10)$ were included in the multivariate analysis. All statistical calculations were performed with SPSS 13.0 for Windows (SPSS, Chicago, IL, USA). P<0.05 was considered to indicate a statistically significant difference.

\section{Results}

Patients' characteristics. A total of 510 NSCLC patients were enrolled in the present study. The characteristics of the patients are summarized in Table I. According to the criteria of the World Health Organization/International Association for the Study of Lung Cancer (WHO/IASLC) classification of lung tumors, 253 tumors were squamous cell carcinomas, 25 were large cell lung carcinomas and 232 were adenocarcinomas; of these, 29 were well differentiated, 263 were moderately differentiated and 218 were poorly differentiated. There were 354 patients who had smoked at a some time in their lives. In terms of the new IASLC staging system, 234 cases were categorized as stage I, 128 as stage II and 148 as stage III.

Platelet count and patients' characteristics. The median platelet count in the NSCLC patients was $203 \times 10^{9} / 1$ (95\% CI, $\left.115-358 \times 10^{9} / 1\right)$. A total of $449(88.0 \%)$ patients had a platelet count of $\leq 300 \times 10^{9} / 1$, which was defined as within the normal range. The correlation between the characteristics of the patients and their platelet count is shown in Table I. There was no significant correlation between the platelet count and gender, age, smoking status, tumor histological type or cancer cell differentiation. The median platelet count in the $\mathrm{N} 2$ patients was significantly higher than that in the $\mathrm{N} 1$ or N0 patients (median, $221 \times 10^{9} / 1$ vs. $217 \times 10^{9} / 1$ or $192 \times 10^{9} / 1$, respectively; $\mathrm{P}=0.001)$. There was a statistically significant correlation between the platelet count and the $\mathrm{T}$ and clinical stages. The median platelet count in the T3 and T4 patients was significantly higher than that in the $\mathrm{T} 1$ and $\mathrm{T} 2$ patients (median, 263 and $253.5 \times 10^{9} / 1$ vs. 199.5 and $196.5 \times 10^{9} / 1$, respectively; $\mathrm{P}<0.001)$. When the value of the platelet count was analyzed as a dichotomous variable (elevated and normal platelet count groups), the frequency of the patients with T3 and T4 was higher in the group that had an elevated platelet count. The increased platelet count was also associated with the $\mathrm{N}$ and clinical stages $(\mathrm{P}<0.05)$.

Association of platelet count with DFS and OS. Overall, the 3-year DFS and OS probabilities were 57.3 and $75.7 \%$, respectively. The median DFS period was 34.0 months in the patients with a normal platelet count and 27.4 months in the patients with an elevated platelet count. The 3-year cumulative OS probability was $75.3 \%$ for patients with a normal platelet count and $59.2 \%$ for patients with an elevated platelet count. The Kaplan-Meier DFS and OS curves of the normal versus elevated platelet counts showed a highly significant separation, as shown in Fig. 1. When stratified by gender, age, smoking status, tumor histological type, tumor differentiation and the $\mathrm{T}, \mathrm{N}$ and clinical stages in each subgroup, the patients with a normal platelet count had a longer mean DFS time than those with an elevated platelet count. Within the subgroups of age $<65$ years, female, poor differentiation, T2 stage, N0 stage and clinical stage I in 
particular , the Kaplan-Meier DFS curves of the normal versus elevated platelet counts showed a significant separation.

In the multivariate survival analysis, the platelet count and patient age, but not the smoking status, tumor differentiation or the T, N and clinical stages, were associated with DFS and OS (Table II).

\section{Discussion}

The results of the present study indicated that the pre-operative platelet count may be used as a biomarker for predicting the outcome in NSCLC. An elevated platelet count was correlated with a worse prognosis in the NSCLC patients. To the best of our knowledge, this is the largest sample study to reveal a correlation between platelet count and the prognosis of operable NSCLC patients.

In the present study, the platelet count was significantly associated with tumor clinical stage and patient outcome. An elevated platelet count was associated with a worse patient outcome. Moreover, multivariate analysis using a Cox proportional hazards model showed that the pre-operative platelet count was an independent prognostic factor in operable NSCLC patients. The patients with an elevated platelet count had a 1.57-fold greater risk of disease progression than those with a normal fibrinogen level. These findings were consistent with a previous study (18), which were based on relatively small sample sizes. Tomita et al (18) reported that the pre-operative platelet count was a prognostic factor for resectable NSCLC patients. The 5-year survival probabilities of patients with normal or elevated platelet counts were reported as 28.87 and $63.73 \%$, respectively. The major strengths of the present study are the inclusion of a large population of NSCLC patients, which was used to investigate the prognostic value of the platelet count. The large size of the study may avoid bias and heterogeneity. However, the present study was also a retrospective study and there was insufficient information on post-recurrence treatment, which may have lead to differences in the survival rates. A prospective study is required to determine the prognostic and treatment value of serum fibrinogen.

Platelets play various significant roles in physiological pathways, including homeostasis and inflammation. Also, platelets correlate with the progression of malignancies. The precise reason for the association between an elevated platelet count and a worse outcome for NSCLC remains unknown. Firstly, the increase in platelet count may promote tumor cell growth and angiogenesis. Platelets release various cytokines, including vascular endothelial growth factor (VEGF) and platelet-derived growth factor (PDGF), during blood clotting. The VEGF and PDGF family of proteins has a significant role in regulating angiogenesis. The invasiveness of the cancer cells may be enhanced by the plasma components in stored platelets (19). Additionally, bevacizumab, an inhibitor of VEGF, is able to reduce this promotive effect. Moreover, platelets promote the formation of capillary-like structures by endothelial cells, via integrins mediating cell-cell adhesion (20). Secondly, platelets enhance tumor metastasis by protecting the tumor cells from the host's immune system. Platelets expressing immunoregulatory proteins, including glucocorticoid-induced TNFR-related (GITR) protein, may protect the cancer cells (21). The inhibition of platelet activa- tion significantly decreases the metastatic potential of tumor cells (22).

In summary, there is evolving evidence that platelet counts are an independent new prognostic biomarker for DFS and OS in operable NSCLC. An assessment of the platelet count should be included in the work up of patients with NSCLC in future prospective trials to confirm its prognostic significance.

\section{References}

1. Siegel R, Naishadham D and Jemal A: Cancer statistics, 2012. CA Cancer J Clin 62: 10-29, 2012.

2. O'Byrne KJ, Gatzemeier U, Bondarenko I, et al: Molecular biomarkers in non-small-cell lung cancer: a retrospective analysis of data from the phase 3 FLEX study. Lancet Oncol 12: 795-805, 2011.

3. Douillard JY, Shepherd FA, Hirsh V, et al: Molecular predictors of outcome with gefitinib and docetaxel in previously treated non-small-cell lung cancer: data from the randomized phase III INTEREST trial. J Clin Oncol 28: 744-752, 2010.

4. Komurcuoglu B, Ulusoy S, Gayaf M, et al: Prognostic value of plasma D-dimer levels in lung carcinoma. Tumori 97: 743-748, 2011.

5. Unsal E, Atalay F, Atikcan S and Yilmaz A: Prognostic significance of hemostatic parameters in patients with lung cancer. Respir Med 98: 93-98, 2004.

6. van Doormaal FF, Raskob GE, Davidson BL, et al: Treatment of venous thromboembolism in patients with cancer: subgroup analysis of the Matisse clinical trials. Thromb Haemost 101: 762-769, 2009.

7. Monreal M, Fernandez-Llamazares J, Piñol M, et al: Platelet count and survival in patients with colorectal cancer - a preliminary study. Thromb Haemost 79: 916-918, 1998.

8. Costantini V, Zacharski LR, Moritz TE and Edwards RL: The platelet count in carcinoma of the lung and colon. Thromb Haemost 64: 501-505, 1990.

9. Shimada H, Oohira G, Okazumi S, et al: Thrombocytosis associated with poor prognosis in patients with esophageal carcinoma. J Am Coll Surg 198: 737-741, 2004.

10. Ikeda M, Furukawa H, Imamura $\mathrm{H}$, et al: Poor prognosis associated with thrombocytosis in patients with gastric cancer. Ann Surg Oncol 9: 287-291, 2002.

11. Hwang SG, Kim KM, Cheong JH, et al: Impact of pretreatment thrombocytosis on blood-borne metastasis and prognosis of gastric cancer. Eur J Surg Oncol 38: 562-567, 2012.

12. Gonzalez Barcala FJ, Garcia Prim JM, Moldes Rodriguez M, et al: Platelet count: association with prognosis in lung cancer. Med Oncol 27: 357-362, 2010.

13. Gislason T and Nõu E: Sedimentation rate, leucocytes, platelet count and haemoglobin in bronchial carcinoma: an epidemiological study. Eur J Respir Dis 66: 141-146, 1985.

14. Engan T and Hannisdal E: Blood analyses as prognostic factors in primary lung cancer. Acta Oncol 29: 151-154, 1990.

15. Pedersen LM and Milman N: Prognostic significance of thrombocytosis in patients with primary lung cancer. Eur Respir J 9: 1826-1830, 1996.

16. Aoe K, Hiraki A, Ueoka H, et al: Thrombocytosis as a useful prognostic indicator in patients with lung cancer. Respiration 71: 170-173, 2004.

17. Cox G, Walker RA, Andi A, et al: Prognostic significance of platelet and microvessel counts in operable non-small cell lung cancer. Lung Cancer 29: 169-177, 2000.

18. Tomita M, Shimizu T, Hara M, et al: Prognostic impact of thrombocytosis in resectable non-small cell lung cancer. Interact Cardiovasc Thorac Surg 7: 613-615, 2008.

19. Dineen SP, Roland CL, Toombs JE, et al: The acellular fraction of stored platelets promotes tumor cell invasion. J Surg Res 153: 132-137, 2009.

20. Pipili-Synetos E, Papadimitriou E and Maragoudakis ME: Evidence that platelets promote tube formation by endothelial cells on matrigel. Br J Pharmacol 125: 1252-1257, 1998.

21. Placke T, Kopp HG and Salih HR: Modulation of natural killer cell anti-tumor reactivity by platelets. J Innate Immun 3: 374-382, 2011.

22. Nieswandt B, Hafner M, Echtenacher B and Männel DN: Lysis of tumor cells by natural killer cells in mice is impeded by platelets. Cancer Res 59: 1295-1300, 1999. 